\section{Surgeons and robots}

\author{
Andreas F. Mavrogenis ${ }^{1} \cdot$ Marius M. Scarlat $^{2}$
}

Published online: 8 May 2019

(C) SICOT aisbl 2019

The Orthopaedic Surgeon is considered a precise individual. The General Orthopaedic Surgeon is educated to have a scientific background, thinks logically and performs surgery with accuracy in a Modern World. There are highly expectations from the patients. However, the surgeon's accuracy could be compared in the near future to robots or machines that would perform the same procedure without error and with higher precision and repeatability. Never tired. Always ready to work. Always precise. No feelings. Only results. Are those machines accurate enough? Is the liability of a robot covered by the hospital or the consultant surgeon would play a role in defining this feature? Computer-assisted navigation and robotic surgery, once considered a science-fiction movie, has evolved and spread rapidly over the last three decades. Currently, for some types of operations it is becoming the standard procedure. The aim for the surgeons using this intelligent software and mechanical arms is to gain access to previously inaccessible areas, and to reduce the risks associated with conventional surgery. The conception for the patients opting for this high-tech solution is to obtain smaller incisions and scars, and to experience faster recovery, reduced post-care discomfort and shorter hospital stay. However, there is a lot of controversy for computer-assisted navigation and robotic systems to be seen as a return of the investment for improved surgical outcomes. Additionally, surgeons must also be trained to use this equipment prior to implementation, and must undergo a learning curve to reduce operative time when using navigation and robotics.

Marius M. Scarlat

mscarlat@gmail.com

1 First Department of Orthopaedics, National and Kapodistrian University of Athens, School of Medicine, Athens, Greece

2 Clinique St. Michel, Group ELSAN, Toulon, France

\section{The evolution}

It appears that the future has arrived for computer-assisted surgery. However, the human element still remains an unpredictable factor, as even small errors may compromise the final result. Additionally, intraoperative imaging and surgical time is increased, and the cost of this technology still is deemed exceptionally high. Moreover, as with all new technology, there is a transition adaptation period. Smartphones are a good example as one of the biggest invention in mankind; in contrast to original LAN phones, current smartphones may be used for communication, socialization, business and bibliometric analyses [1]. Computer-assisted surgery can be either navigation or robotics; the two systems are completely different.

Computer-assisted navigation systems use referencing methods to virtually reconstruct the area of interest and display it on a screen enabling the surgeon to receive real-time feedback intraoperatively [2]. Robotics use computer-guided mechanical arms that enable surgeons to perform surgery remotely, and allow for more accurate and reproducible placement of surgical instruments and implants [3]. Based on the method used for information referencing, navigation systems can be computer tomography (CT)-based, fluoroscopy-based and imageless. CT-based systems require a CT scan of the area, performed either pre- or intraoperatively with a specific protocol in order to reconstruct a three-dimensional image of the surgical field. The surgeon can either virtually plan his operation beforehand and match his actions during the procedure, or use the navigation system in real-time mode, allowing him to check the position of his instruments and implants at any given time during the procedure. Fluoroscopy-based systems require the positioning of a certain amount of markers on specific anatomical landmarks, which are then captured by a series of fluoroscopic images. The surgeon can then relate the position of his instruments in space. Imageless systems require an optical camera and infrared markers. They rely on the surgeon to pinpoint certain stable predefined landmarks 
with the use of the markers and determine the centre of joint rotation with the use of kinematics [2]. Based on the surgeons' feedback to the procedure, robotic systems can be passive, semi-active, and active. Passive systems guide surgeons who direct the robotic instrumentation to perform a task. Semiactive systems constrain surgical manipulation through feedback to restrict what can be done surgically. Active systems may independently perform tasks without direct human manipulation and surgeons' control intraoperatively. Robots can act directly, when they cut the bone to the final desired shape; or indirectly, when they machine features in the bone to allow placement of cutting jigs. Furthermore, robotic bone cutting can be autonomous, when the robot cuts bone with no controlling human hand; haptic, when human interaction is required to move the robot to cut but the robot's movement is constrained by a border; and boundary, when human interaction is required to move the robot but cutting is deactivated or prevented by some means if it travels beyond a boundary even though it is free to move anywhere in space [3].

\section{The clinical applications}

Computer-assisted navigation and robotics are currently being used in many orthopaedic procedures and multiple orthopaedic subspecialties. These include total joint arthroplasties, arthroscopic surgery, spine, tumor and fracture surgery. With the use of navigation systems in total joint arthroplasties, the implants are better aligned, the knee joint can be more accurately balanced, and soft-tissues release can be more accurately performed; although it remains controversial, blood loss and intraoperative fat embolism can also be reduced. Robotic systems have been used successfully in unicompartmental knee arthroplasty, patellofemoral arthroplasty, and total knee arthroplasty. With the use of semi-active robotic systems in total hip arthroplasty, the surgeon may control the robotic arm to ream the acetabulum to a specified depth and size, without the need for sequential reaming. Imaging software can also control for limb length equality [3-11].

The application of robotic systems in spinal surgery has facilitated screw placement in spinal fusions, tumor resection, and revision operations. However, there is insufficitn evidence to recommend robotics versus conventional techniques in spinal surgery [3]. Navigation systems can be helpful in fracture treatment especially with minimal invasive techniques, such as percutaneous fixation of femoral head fractures with cannulated screws, fractures of the acetabulum, the sacroiliac joint, the tibial plateau and the humeral head; they may assist the surgeon to determine the entry point and angle of insertion of the guide wires, and to restore normal limb rotation [2]. Robotic systems have assisted with planning closed fracture reduction and reconstruction and in performing surgery remotely such as in telemedicine in small towns, rural areas, and war zones $[3,12]$. Oncologic reconstruction using robotic made implants is already performed with good results [13].

\section{The concerns}

Certain surgical cases should not be done with navigation or robotic systems because there is no evidence to support the increased cost and effectiveness over conventional techniques. Additionally, security issues and vulnerability of computerassisted surgical systems to hacking and hijacking is a major concern; strong passwords and effective firewalls are necessary in this respect. Concerns are more pronounced for robotic systems that make use of artificial intelligence. Artificial intelligence may analyze complex medical data and assist physicians as intelligent assistants. Using artificial intelligence, fully autonomous robotic systems may be used to perform operations in areas that remote surgery is not always possible, such as in the space. Research is ongoing to find the better tactile feedback of a robotic system to a patient that would be similar to a surgeon's hands.

\section{The future}

Computer-assisted surgery will undoubtfully play an important role in surgical practice in the future. Robotic systems are more likely to be autonomous; this is expected to reduce the role of the surgeons, however, computer-assisted surgery is considered safer than open surgery, it is unclear how the tactile feedback of robots would be accepted by the patients because completely replacing surgeons with autonomous active robots may entail risks and complications. Robotic systems may also be connected to the internet as a group, to learn from one another with the information obtained from one robot saved and transmitted to the interconnected. Artificial intelligence may provide for intelligent assistants. Postoperatively, rehabilitation robots may support the patients, improve movement and generate the appropriate rehabilitation protocols. Disadvantages related to increased cost and surgical time are expected to be solved, and more data will report whether the use of robotics can improve patient clinical outcomes. If not, the use of computer-assisted orthopaedic surgery will be short.

\section{References}

1. Scarlat MM, Mavrogenis AF, Pećina M, Niculescu M (2015) Impact and alternative metrics for medical publishing: our experience with International Orthopaedics. Int Orthop 39(8):1459-1464. https://doi.org/10.1007/s00264-015-2766-y

2. Mavrogenis AF, Savvidou OD, Mimidis G, Papanastasiou J, Koulalis D, Demertzis N, Papagelopoulos PJ (2013) Computer- 
assisted navigation in orthopedic surgery. Orthopedics 36(8):631642

3. Chen AF, Kazarian GS, Jessop GW, Makhdom A (2018) Robotic Technology in Orthopaedic Surgery. J Bone Joint Surg Am 100(22):1984-1992

4. Turktas U, Piskin A, Poehling GG (2016) Short-term outcomes of robotically assisted patello-femoral arthroplasty. Int Orthop 40(5): 919-924. https://doi.org/10.1007/s00264-015-2786-7

5. Putzer D, Moctezuma JL, Nogler M (2017) Computer aided planning of orthopaedic surgeries: the definition of generic planning steps for bone removal procedures. Int Orthop 41(11):2221-2227. https://doi.org/10.1007/s00264-017-3626-8

6. Cavinatto L, Bronson MJ, Chen DD, Moucha CS (2018) Roboticassisted versus standard unicompartmental knee arthroplastyevaluation of manuscript conflict of interests, funding, scientific quality and bibliometrics. Int Orthop. https://doi.org/10.1007/ s00264-018-4175-5

7. Cho KJ, Seon JK, Jang WY, Park CG, Song EK (2018) Robotic versus conventional primary total knee arthroplasty: clinical and radiological long-term results with a minimum follow-up of ten years. Int Orthop. https://doi.org/10.1007/s00264-018-4231-1

8. Herry Y, Batailler C, Lording T, Servien E, Neyret P, Lustig S (2017) Improved joint-line restitution in unicompartmental knee arthroplasty using a robotic-assisted surgical technique. Int Orthop 41(11):2265-2271. https://doi.org/10.1007/s00264-017-3633-9

9. Karunaratne S, Duan M, Pappas E, Fritsch B, Boyle R, Gupta S, Stalley P, Horsley M, Steffens D (2018) The effectiveness of robotic hip and knee arthroplasty on patient-reported outcomes: a systematic review and meta-analysis. Int Orthop. https://doi.org/10.1007/ s00264-018-4140-3

10. Matsumoto T, Nakano N, Lawrence JE, Khanduja V (2018) Current concepts and future perspectives in computer-assisted navigated total knee replacement. Int Orthop. https://doi.org/10.1007/ s00264-018-3950-7

11. d'Amato M, Ensini A, Leardini A, Barbadoro P, Illuminati A, Belvedere C (2018) Conventional versus computer-assisted surgery in total knee arthroplasty: comparison at ten years follow-up. Int Orthop. https://doi.org/10.1007/s00264-018-4114-5

12. Qiao F, Li D, Jin Z, Hao D, Liao Y, Gong S (2016) A novel combination of computer-assisted reduction technique and three dimensional printed patient-specific external fixator for treatment of tibial fractures. Int Orthop 40(4):835-841. https://doi.org/10.1007/ s00264-015-2943-z

13. Angelini A, Trovarelli G, Berizzi A, Pala E, Breda A, Ruggieri P (2019) Three-dimension-printed custom-made prosthetic reconstructions: from revision surgery to oncologic reconstructions. Int Orthop 43(1):123-132. https://doi.org/10.1007/s00264-0184232-0

Publisher's note Springer Nature remains neutral with regard to jurisdictional claims in published maps and institutional affiliations. 